\title{
Logarithmic transformation technique for exact signal recovery in frequency-domain optical-coherence tomography
}

\author{
S. Chandra Sekhar ${ }^{a}$, Rainer A. Leitgeb ${ }^{b}$, Adrian H. Bachmann ${ }^{c}$, and Michael Unser ${ }^{d}$ \\ a,d Laboratoire d'imagerie biomédicale, ${ }^{b, c}$ Laboratoire d'optique biomédicale \\ École polytechnique fédérale de Lausanne (EPFL), Switzerland
}

\begin{abstract}
We address the problem of exact signal recovery in frequency-domain optical-coherence tomography (FDOCT). The standard technique for tomogram reconstruction is the inverse Fourier transform. However, the inverse Fourier transform is known to yield autocorrelation artifacts which interfere with the desired signal. We propose a new transformation for computing an artifact-free tomogram from intensity measurements. Our technique relies on the fact that, in the FDOCT measurements, the intensity of the total signal reflected from the object is smaller than that of the reference arm. Our technique is noniterative, nonlinear, and it leads to an exact solution in the absence of noise. The reconstructed signal is free from autocorrelation artifacts. We present results on synthesized data as well as on experimental FDOCT measurements of the retina of the eye.
\end{abstract}

Keywords: frequency-domain optical-coherence tomography, autocorrelation artifacts.

\section{INTRODUCTION}

Frequency-domain optical-coherence tomography (FDOCT) is an effective and noninvasive interferometric technique that is suitable for three-dimensional imaging of biological specimens. Using FDOCT, one can acquire the information of the depth scan of the specimen within a single exposure. FDOCT is becoming increasingly popular because it is significantly faster than its counterpart-time-domain optical coherence tomography (TDOCT). FDOCT has higher sensitivity and signal-to-noise ratio compared to TDOCT. ${ }^{1,2}$ FDOCT is also amenable to high-resolution imaging by using the principles of phase-shifting interferometry. ${ }^{3}$ The primary applications of FDOCT have been in dermatology ${ }^{4}$ and opthalmology. 5,6

In the FDOCT experiments, the measurements are spectral intensities, from which we desire to construct a tomogram of the object. The commonly-employed technique is the inverse Fourier transform; however, it is known to introduce autocorrelation artifacts that can seriously degrade the resolution of the tomogram. The most-advanced method to date uses minimum-phase functions and provides an iterative reconstruction. ${ }^{7}$ This algorithm is similar to the Fienup iterative algorithm ${ }^{8,9}$ with a causality constraint in the spatial domain.

In this paper, we propose a new noniterative technique that enables exact signal recovery. The technique completely eliminates autocorrelation artifacts and produces interference-free tomograms.

\footnotetext{
Contact information:

$a, d$ Tel: +41 216935135 ; Emails: $\left\{{ }^{a}\right.$ chandrasekhar.seelamantula, $d_{\text {michael.unser }\} @ e p f l . c h}$

b, ${ }^{c}$ Tel: +41216935726 ; Emails: $\left\{{ }^{b}\right.$ rainer.leitgeb, ${ }^{c}$ adrian.bachmann $\} @$ epfl.ch
}

Optical Coherence Tomography and Coherence Techniques III, edited by Peter E. Andersen, Zhongping Chen, Proc. of SPIE-OSA Biomedical Optics, SPIE Vol. 6627, 662714, @ 2007 SPIE-OSA - 1605-7422/07/\$18 


\section{SIGNAL ACQUISITION}

In Fig. 1, we show a schematic of the FDOCT data-acquisition setup. A broadband source is used for illumination. The output of the source is optically split into two beams which are directed towards the two arms of a Michelson interferometer:

1. A broadband reference mirror is placed at the end of the reference arm. The light reflected from the broadband mirror is the reference signal.

2. The specimen to be imaged is placed at the end of the object arm. The object largely absorbs, scatters, and attenuates the incident light; only a small fraction is reflected back. Therefore, the reflected light intensity is small compared to that of the reference.

The two reflected signals are combined in a collinear fashion and directed to an optical spectrum analyzer which performs a spectral decomposition of the signal as a function of the wavelength $\lambda$. Since the light from the object is weak compared to that from the reference, it manifests itself as a spectral fringe riding on top of the source spectrum. The offset distance $z_{0}$ between the zero-phase delay plane and the surface of the object (see Fig.1) is always positive in our experimental setup.

For volume-imaging applications, the specimen is scanned in the lateral direction. The measurements are processed on a scan-by-scan basis. The scattering functions recovered from all the scans are put together to form the tomogram.

\section{SIGNAL MODEL}

The measured output of the optical spectrum analyzer is a function of the wavelength and it is mapped onto the wavenumber scale $k$. The resulting interference signal $I(k)$ is given by the following equation (see the chapter by Lindner et.al. ${ }^{10}$ for details):

$$
I(k)=S(k)\left|1+\int_{0}^{\infty} a(z) e^{j 2 k n(z) z} d z\right|^{2},
$$

where

$n(z)$ is the refractive index as a function of the depth $z$,

$a(z)$ is the amplitude of the wave backscattered from the object, and

$S(k)$ is the spectrum intensity distribution of the broadband light source for a given wavenumber.

We ignore the dispersion in the specimen. We also make the zeroth-order approximation $n(z)=n$. This assumption is widely used and holds when the bandwidth of the source is moderate. We also make the substitution $\omega=-2 k n$, so that

$$
I(\omega)=S(\omega)\left|1+\int_{0}^{\infty} a(z) e^{-j \omega z} d z\right|^{2} .
$$

The tomogram-reconstruction problem now simplifies to recovering $a(z)$ given $I(\omega)$. The source spectrum $S(\omega)$ can be measured by blocking the object arm. Therefore, the term of interest is

$$
H(\omega)=\left|1+\int_{0}^{\infty} a(z) e^{-j \omega z} d z\right|^{2}
$$

which is the measurement normalized with respect to the source spectrum. 


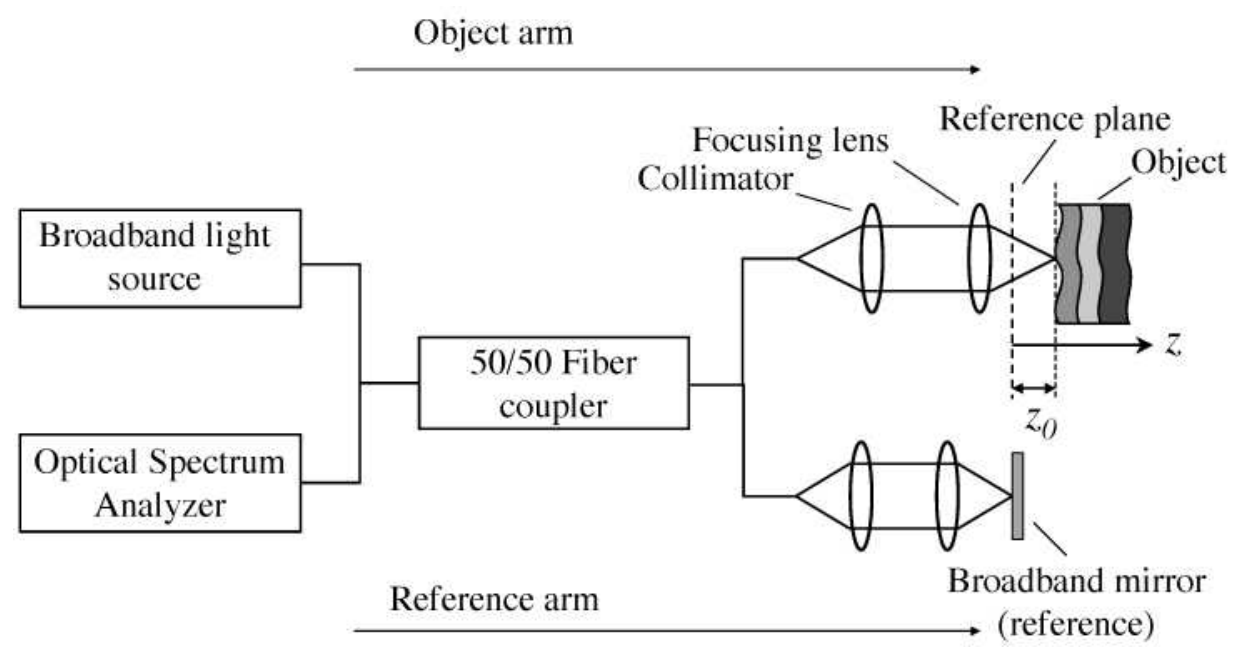

Figure 1. Schematic of the Fourier-domain optical-coherence tomography system.

\section{AUTOCORRELATION ARTIFACTS}

Define $A(\omega)=\int_{0}^{\infty} a(z) e^{-j \omega z} d z$. Developing the squares in (3), we get

$$
H(\omega)=1+|A(\omega)|^{2}+A(\omega)+A^{*}(\omega) .
$$

Taking the inverse Fourier transform of both sides of (4), we get

$$
h(z)=\delta(z)+r_{a a}(z)+a(z)+a(-z),
$$

where $a(z)$ is the desired term and where $a(-z)$ is its mirror image. $r_{a a}(z)$ is the autocorrelation term which interferes with $a(z)$ and $a(-z)$ thereby causing artifacts in the tomogram. If $z_{0}>0, a(z)=0$ for $z<z_{0}$ and does not overlap with its mirror image $a(-z)$.

\section{LOGARITHMIC TRANSFORMATION TECHNIQUE}

The intensity of the signal reflected from the object is much smaller than the reference intensity. Therefore, we have that $|A(\omega)| \ll 1, \forall \omega$. The autocorrelation artifact is the result of the multiplicative interaction between $A(\omega)$ and its conjugate. To convert the multiplicative interaction to an additive one, let us consider the logarithm of $H(\omega)$ :

$$
\begin{aligned}
\log (H(\omega)) & =\log (1+A(\omega))+\log \left(1+A^{*}(\omega)\right) \\
& =A(\omega)+A^{*}(\omega)+\mathcal{O}\left(A^{2}(\omega)\right)+\mathcal{O}\left(A^{* 2}(\omega)\right),
\end{aligned}
$$

where $\mathcal{O}$ denotes the Landau symbol. The second equality above has been obtained by using a Taylor series expansion under the hypothesis that $|A(\omega)| \ll 1$.

We note that $A(\omega)$ has a causal inverse. Therefore, $A^{n}(\omega)$ also has a causal inverse for $n \geq 1$. Similarly, $A(\omega)$ and $A^{* n}(\omega)$ have non-causal inverses. Thus, the logarithm neatly separates the signal into causal and non-causal parts. Retaining the causal part in the inverse of $\log (H(\omega))$ and applying a Fourier transform, we get $\log (1+A(\omega))$. $A(\omega)$ can be recovered from $\log (1+A(\omega))$ by complex exponentiation. The desired function $a(z)$ can be computed from $A(\omega)$ by inverse Fourier transformation. 


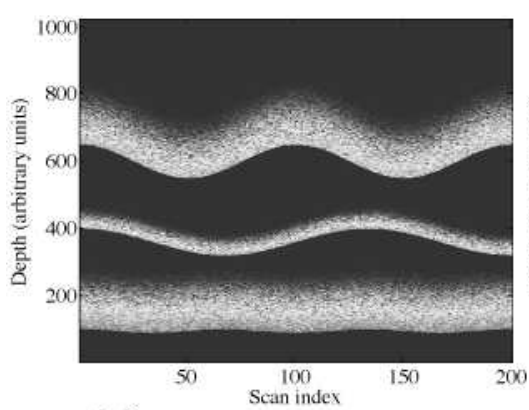

(a)

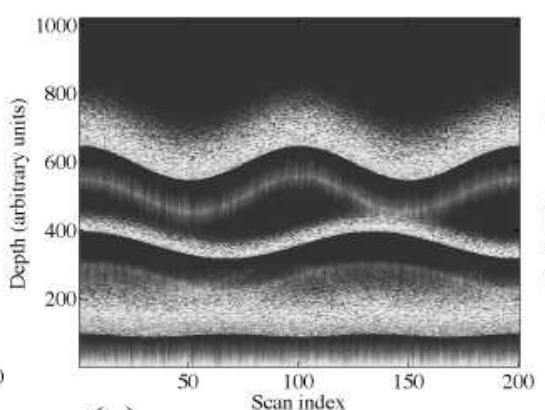

(b)

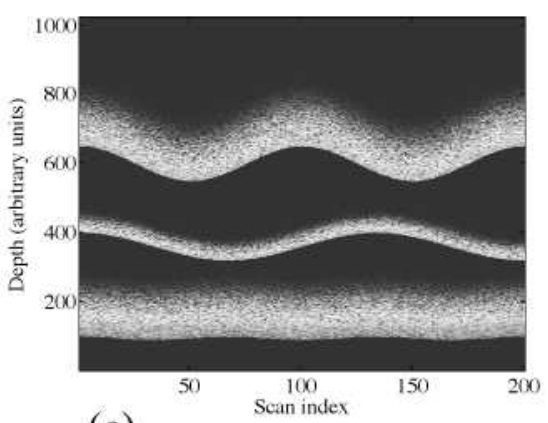

(c)

Figure 2. (a) The synthesized scattering function of a three-layer specimen, (b) The scattering function recovered by the inverse Fourier transform, (c) The scattering function obtained by using the logarithmic-transformation technique.

Thus, the logarithmic transformation enables exact recovery of $a(z)$ from $H(\omega)$.

In practice, the Fourier transform can be implemented by using the fast-Fourier-transform (FFT) algorithm. The logarithm and exponential operations are nonlinear; therefore, oversampling is necessary to suppress aliasing errors. Typically, oversampling by a factor of two suffices for practical applications.

\section{EXPERIMENTAL RESULTS}

We validate our technique on both synthesized and experimental data. We synthesized a multilayer biological specimen with a scattering function as shown in Fig. 2(a). We assume that the source power spectrum is perfectly flat. The tomogram constructed by using the standard inverse-Fourier-transform technique is shown in Fig. 2(b). Note that autocorrelation artifacts interfere with the structure of the specimen. The scattering function recovered by using the logarithmic transformation is shown in Fig. 2(c). Note that the autocorrelation artifacts have been completely suppressed.

Next, we report the performance on FDOCT measurements of the human-eye retina. The experimental setup is shown in Fig. 3. The broadband light source is synthesized by using two superluminescent diodes (EXALOS) with center wavelengths $853 \mathrm{~nm}$ and $827 \mathrm{~nm}$. The respective full-width-athalf-maximum values (FWHM) are $34 \mathrm{~nm}$ and $25 \mathrm{~nm}$, resulting in an overall FWHM of $36 \mathrm{~nm}$, a central wavelength of $833.5 \mathrm{~nm}$, and an axial resolution of $8.5 \mu \mathrm{m}$ in air. The dispersion compensation in the reference arm matches the sample-arm optics as well as the water chamber of the eye. The light in the sample path passes an X-Y galvo scanning stage (Cambridge Technology) and illuminates the eye via a telescope with angular magnification of two. At the cornea, the optical power is $300 \mu \mathrm{W}$ and the beam waist is $1.8 \mathrm{~mm}$ which results in a theoretical spot size of $14 \mu \mathrm{m}$ at the retina. The recombined reference and sample-arm light is guided through a single-mode fiber to the spectrometer module. The latter is equipped with a volume-transmission-diffraction grating (Wasatch, 1200lines $/ \mathrm{mm}$ ) and an objective lens with focal length of $135 \mathrm{~mm}$. The CCD line-scan camera (ATMEL AVIIVA, 2048 pixel, 12bit) is driven at a line rate of $17.4 \mathrm{kHz}$ with an integration time of $43 \mu \mathrm{s}$. The measured sensitivity is $98 \mathrm{~dB}$ close to the zero-offset distance.

We processed the acquired data by both techniques. In the standard inverse-Fourier-transform technique, it is common practice to suppress the source (background) term by subtraction, so as to increase contrast. The resulting image is shown in Fig. 4(a). The tomogram obtained with the logarithmictransformation technique described in Sec. 5 is shown in Fig. 4 (b). Note that the artifacts have been 


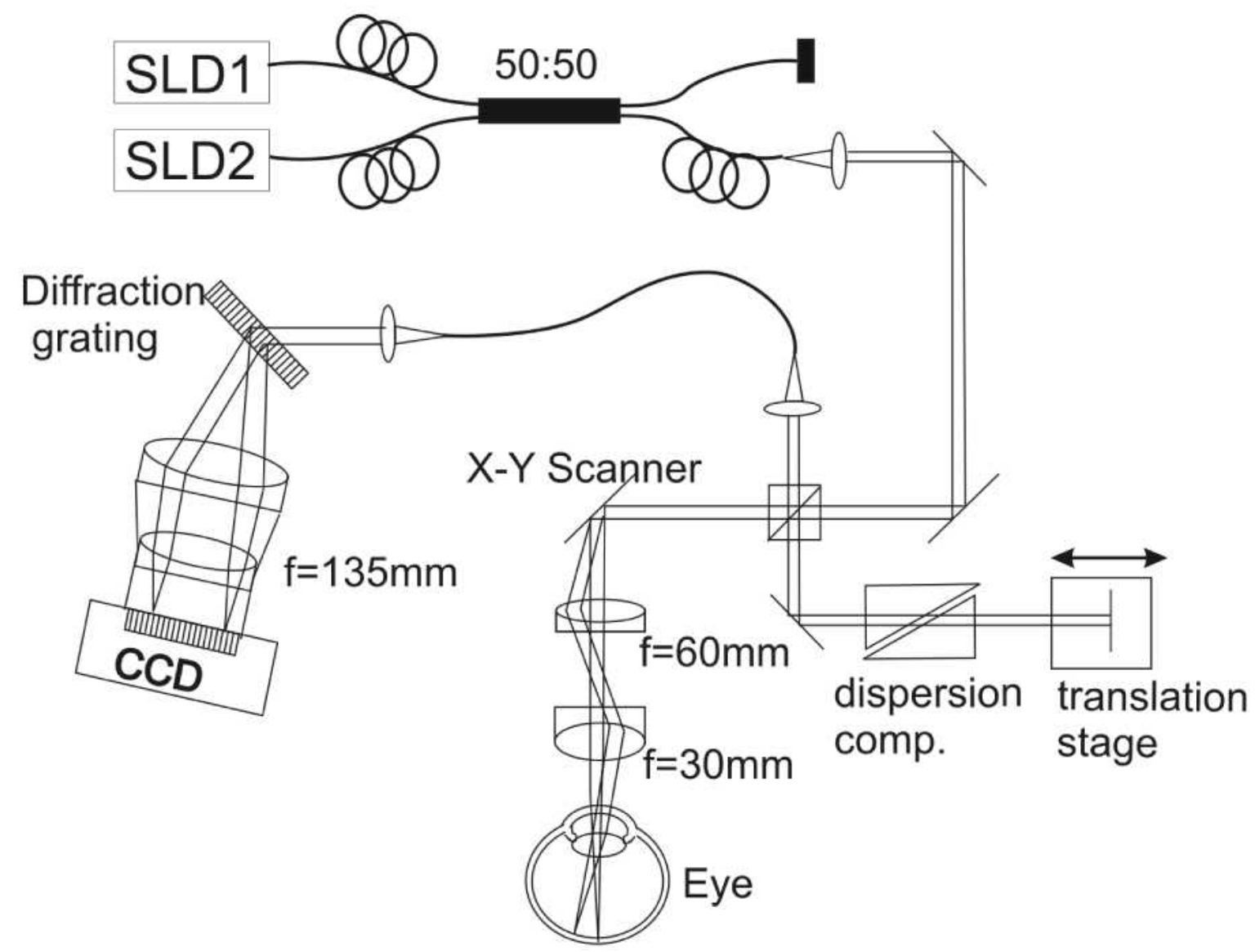

Figure 3. FDOCT experimental setup. SLD stands for superluminescent diode, CCD stands for charge-coupleddevice camera.

suppressed and that the structure of the specimen is also neatly resolved. Since the CCD camera had a noise floor of $40 \mathrm{~dB}$, we retained only the top $40 \mathrm{~dB}$ for display purposes. The two tomograms were also adjusted to the same dynamic range.

\section{CONCLUSION}

We proposed a new technique for exact signal recovery in a FDOCT system. The technique is noniterative, nonlinear, and offers an exact solution in the absence of noise. It is also computationally more efficient than the iterative techniques reported in the literature. Simulation results on synthesized and experimental data showed that the technique offers superior quality and artifact-free reconstruction. The technique can also be extended to higher dimensions which may be useful for holographic imaging applications. ${ }^{11,12}$

\section{REFERENCES}

1. R. Leitgeb, C. K. Hitzenberger and A. F. Fercher, "Performance of Fourier domain vs. time domain optical coherence tomography", Opt. Express, Vol. 11, No. 8, pp. 889-894, 21 April 2003. 

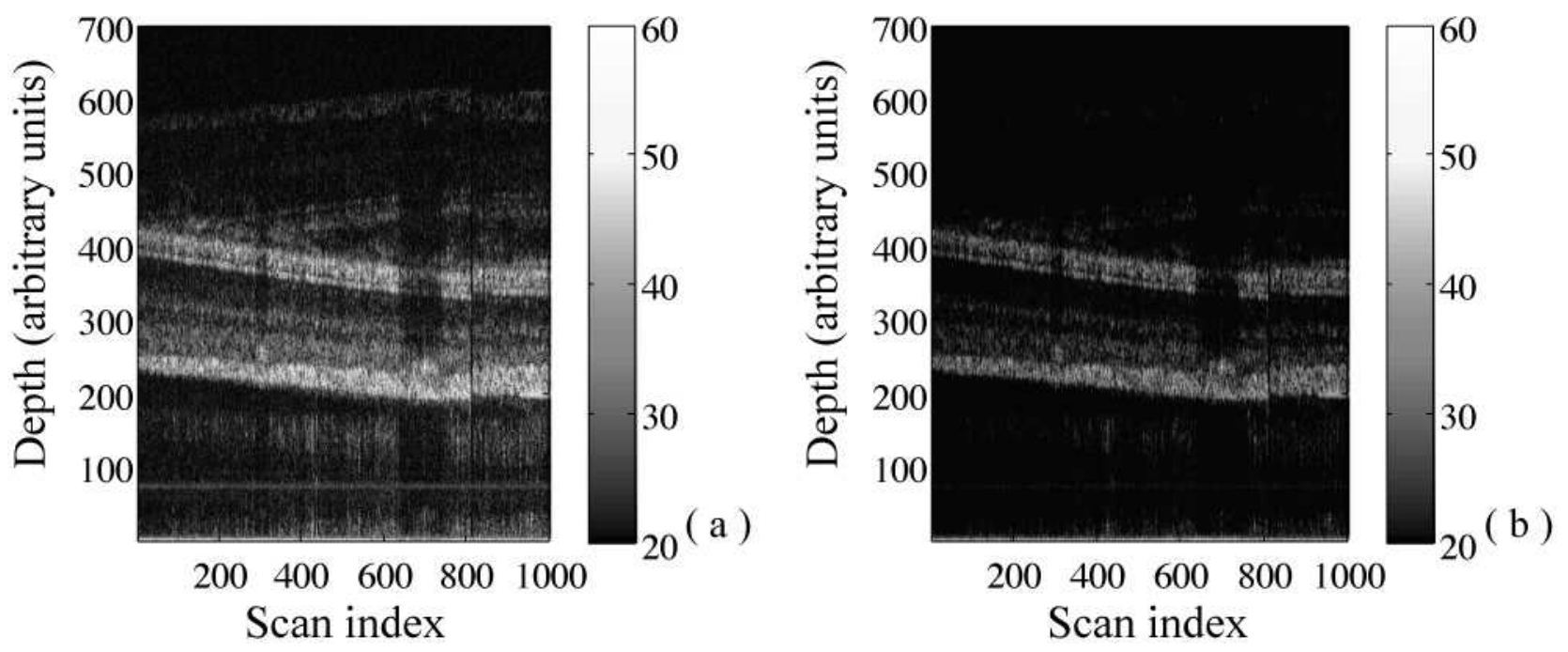

Figure 4. (a) Tomogram obtained with background subtraction and inverse-Fourier-transform, and (b) Tomogram obtained by using the logarithmic-transformation technique.

2. J. F. de Boer, B. Cense, B. H. Park, M. C. Pierce, G. J. Tearney, and B. E. Bouma, "Improved signal-tonoise ratio in spectral-domain compared with time-domain optical coherence tomography" , Opt. Letters, Vol. 28, 2067-2069, 2003.

3. R.A. Leitgeb, C.K. Hitzenberger, A.F. Fercher and T. Bajraszewski, "Phase-shifting algorithm to achieve high-speed long-depth-range probing by frequency domain optical coherence tomography", Opt. Letters, 28, 2201-2203, 2003.

4. G. Hausler, M. W. Lindner, "Coherence radar and spectral radar-new tools for dermatological analysis", Jl. Biomed. Opt., 3, pp.21-31, 1998.

5. M. Wojtkowski, R. Leitgeb, A. Kowalczyk, T. Bajraszewski, A. F. Fercher, "In-vivo human retinal imaging by Fourier domain optical coherence tomography", Jl. Biomed. Opt., 7, pp.457-463, 2002.

6. A. F. Fercher, C. K. Hitzenberger, G. Kamp, S. Y. El-Zaiat, "Measurement of intraocular distances by backscattering spectral interferometry", Opt. Comm., 117, pp. 43-48, 1995.

7. A. Ozcan, M. J. F. Digonnet, G. S. Kino, "Minimum-phase-function-based processing in frequency-domain optical coherence tomography systems", Vol. 23, No. 7, Jl. Opt. Soc. Am. (A), July 2006.

8. J. R. Fienup, "Phase retrieval algorithms: A comparison", Appl. Opt., 21, pp. 2758-2769, 1982.

9. H. H. Bauschke, P. L. Combettes, and D. R. Luke, "Phase retrieval, error reduction algorithm, and Fienup variants: A view from convex optimization", Jl. Opt. Soc. Am., pp. 1334-1345, Vol. 19, Issue 7, July 2002.

10. B.E. Bouma and G.J. Tearney (Ed.), Handbook of optical coherence tomography, Marcel Dekker, Inc., 2002.

11. E. Cuche, "Numerical reconstruction of digital holograms: Application to phase-contrast imaging and microscopy", Doctoral thesis No. 2182, Ecole polytechnique fédérale de Lausanne (EPFL), 2000.

12. M. Liebling, T. Blu, M. Unser, "Complex-wave retrieval from a single off-Axis hologram," Jl. Opt. Soc. Am (A), Vol. 21, No. 3, pp. 367-377, Mar 2004. 